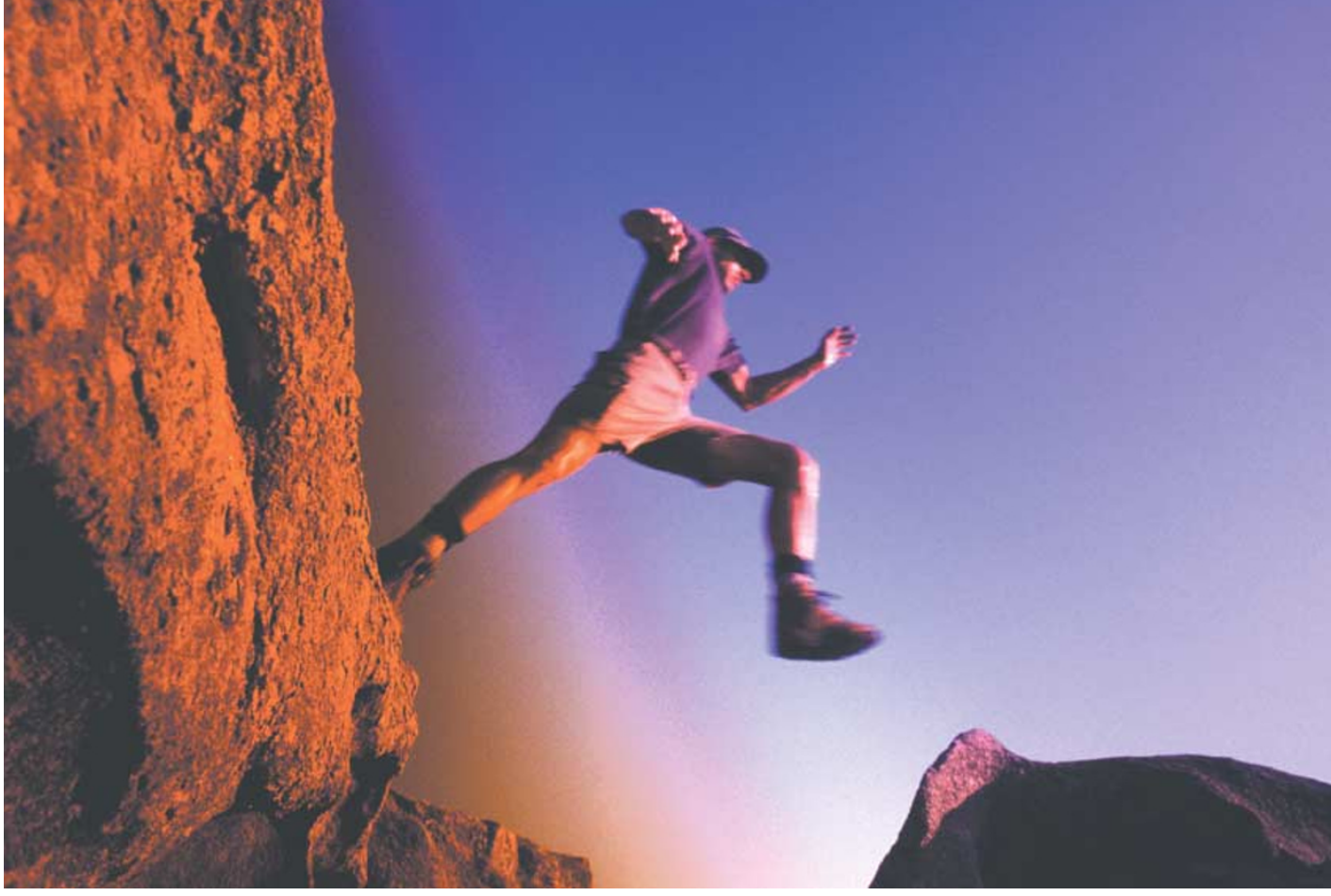

PROTEIN TRANSLOCATION

\section{Crossing the divide}

Most mitochondrial proteins are synthesized in the cytosol, so they reach their final destination courtesy of the TOM complex (translocase of the outer mitochondrial membrane) and one of two TIM complexes (translocases of the inner mitochondrial membrane). The TIM23 complex which is the focus of two recent Cell papers, one from Nikolaus Pfanner's laboratory and one from Toshiya Endo's laboratory — translocates presequence-containing proteins across, or into, the inner mitochondrial membrane (IMM). And, until these recent papers, how these preproteins got from the TOM complex to the TIM23 complex was a mystery.

Both groups began by identifying a new component of the TIM23 complex. Pfanner and colleagues isolated this complex from yeast mitochondria and used mass spectrometry to identify a new $\sim 50 \mathrm{kDa}$ subunit. Endo and co-workers, on the other hand, identified the same subunit by characterizing a protein that they had previously shown could be crosslinked to a translocation intermediate.

The protein identified was called Tim50, and both groups showed that it is essential for the viability of yeast cells. They predicted that is has a single transmembrane domain and found that it is localized to the IMM, where it is positioned so that it exposes its large carboxy-terminal domain to the intermembrane space (IMS). This orientation contrasts with other components of the TIM23 complex, which are either mainly localized in the IMM (for example, Tim23, which forms the channel) or are situated on the matrix side of the IMM (for example, mtHsp70, which is the import motor).

So, how does Tim50 associate with the TIM23 complex? Both groups found that Tim50 binds Tim23, and Endo and colleagues showed that this interaction is dynamic. Furthermore, both groups used yeast two-hybrid analyses to show that the IMS region of Tim50 interacts with residues $\sim 51-96$ of Tim23. This fits well with what is known about Tim23, as these residues stretch across the IMS (the amino-terminal segment of Tim 23 spans the outer mitochondrial membrane).

Tim50 was shown to be directly involved in mitochondrial protein import, as the authors of both studies found that anti-Tim50 antibodies can block the import of preproteins across the IMM. In addition, both groups found that Tim50 can be crosslinked to translocating preproteins, and that it can be purified with a TOM-TIM23-preprotein complex. Endo and colleagues also showed that, when translocation intermediates are inside the TOM channel, they can interact with Tim50 of the TIM23 complex, which indicates that Tim50 has a role in linking translocation through the TOM and TIM23 complexes.

Using galactose-inducible promoters to control the expression of Tim50, both groups found that selective depletion of Tim50 impaired the import of presequence-containing preproteins. However, Pfanner and colleagues also showed that Tim50 depletion only mildly affected the import of preproteins that contain a presequence and an inner-membrane sorting signal. This indicates that Tim50 is needed to import proteins with classical presequences, but that it is not essential to import presequence-containing proteins that also have a sorting signal.

Together, the studies of Pfanner and colleagues and Endo and coworkers have provided insights into how presequence-containing proteins cross the divide between the TOM and TIM23 complexes. The missing link was Tim50, which guides preproteins from the TOM complex across the IMS to Tim23 of the TIM23 complex.

Rachel Smallridge

\section{(0) References and links}

ORIGINAL RESEARCH PAPERS Geissler, A. et al. The mitochondrial presequence translocase: an essential role of Tim50 in directing preproteins to the import channel. Cell 111, 507-518 (2002) | Yamamoto, H. et al. Tim50 is a subunit of the TIM23 complex that links protein translocation across the outer and inner mitochondrial FURTHER READING Neupert, W. \& Brunner, M. The protein import motor of mitochondria. Nature Rev. Mol. Cell Biol. 3, 555-565 (2002) membranes. Cell 111, 519-528 (2002)

\section{IN THE NEWS}

Hearty news

A rapid new NMR-based blood test developed by scientists at Imperial

College, London, and Cambridge University could herald 'The Next Generation' of Star Treklike diagnostic tools.

The technique, published in Nature Medicine (December 2002), could save the lives of thousands of people at risk from heart disease, say researchers.

Requiring only a few drops of blood, the test, called Metabonomics, uses high frequency radio waves to measure the magnetic properties of the molecules.

Metabolites including lipoproteins, lipids and cholines confer a different NMR profile on blood taken from patients with severe atherosclerosis compared with patients without the disease.

As reported in BBC News (24 November), in smallscale trials, Metabonomics "...matched the accuracy of a full angiography [X-ray examination of blood vessels following the injection of a dye into the artery] test in $95 \%$ of cases...", but the noninvasive, simple and cheap nature of the test offers the chance of providing an effective screening service. "It is the closest that science has come so far to the hand-held diagnostic analyser used by Dr McCoy in Star Trek", said Jeremy Nicholson of Imperial College, who was involved in the research.

If larger trials, currently underway at one of Britains's top heart hospitals, Papworth, are successful, the test could be widely available within two years. "Metabonomics has the potential to help detect and diagnose ... clinical problems from bone disease to cancer", Elaine Holmes from Imperial College told Science Daily $(25$ November 2002).

Katrin Bussell 